\title{
Duodenal Fistula, CTCAE
}

National Cancer Institute

\section{Source}

National Cancer Institute. Duodenal Fistula, CT CAE. NCI Thesaurus. Code C57789.

A disorder characterized by an abnormal communication between the duodenum and another organ or anatomic site. 